\title{
Femtosecond dynamics of resonant tunneling and superlattice relaxation in quantum cascade lasers
}

\section{Citation}

Choi, Hyunyong, Theodore B. Norris, Tobias Gresch, Marcella Giovannini, Jérôme Faist, Laurent Diehl, and Federico Capasso. 2008. "Femtosecond Dynamics of Resonant Tunneling and Superlattice Relaxation in Quantum Cascade Lasers." Applied Physics Letters 92 (12): 122114. https://doi.org/10.1063/1.2898518.

\section{Permanent link}

http://nrs.harvard.edu/urn-3:HUL.InstRepos:41372466

\section{Terms of Use}

This article was downloaded from Harvard University's DASH repository, and is made available under the terms and conditions applicable to Open Access Policy Articles, as set forth at http:// nrs.harvard.edu/urn-3:HUL.InstRepos:dash.current.terms-of-use\#OAP

\section{Share Your Story}

The Harvard community has made this article openly available. Please share how this access benefits you. Submit a story.

Accessibility 


\title{
Femtosecond dynamics of resonant tunneling and superlattice relaxation in quantum cascade lasers
}

\author{
Hyunyong Choi, ${ }^{1, a)}$ Theodore B. Norris, ${ }^{1}$ Tobias Gresch, ${ }^{2}$ Marcella Giovannini, ${ }^{2}$ \\ Jérôme Faist, ${ }^{2}$ Laurent Diehl, ${ }^{3}$ and Federico Capasso ${ }^{3}$ \\ ${ }^{1}$ Center for Ultrafast Optical Science and Department of Electrical Engineering and Computer Science, \\ University of Michigan, Ann Arbor, Michigan 48109-2099, USA \\ ${ }^{2}$ Institute of Physics, University of Neuchâtel, CH-2000 Neuchâtel, Switzerland \\ ${ }^{3}$ School of Engineering and Applied Sciences, Harvard University, Cambridge, Massachusetts 02138, USA
}

(Received 29 October 2007; accepted 27 February 2008; published online 28 March 2008)

\begin{abstract}
Time-resolved mid-infrared pump-probe measurements are performed on a quantum cascade laser below and above the threshold. The gain recovery is determined by the electron transport through the cascade heterostructure. Subpicosecond resonant tunneling injection from the injector ground state into the upper lasing state is found to be incoherent due to the strong dephasing in the active subband. The gain recovery due to transport through superlattice is interpreted in terms of dielectric relaxation within the superlattice miniband. (C) 2008 American Institute of Physics.
\end{abstract}

[DOI: 10.1063/1.2898518]

In a quantum cascade laser (QCL), a complex heterostructure controls the tunneling and superlattice relaxation of electrons as they move through the device, enabling a population inversion to be obtained in a unipolar device. Thus, QCL lasing action results from the interplay of the electronic transport through the cascade structure. ${ }^{1}$ Because the laser operates with the electron population far from the equilibrium, it is desirable to investigate the nonequilibrium carrier dynamics directly in the time domain.

Ultrafast optical techniques have been used previously to study transport and relaxation in quantum-well structures relevant to QC operation ${ }^{2-4}$ although very few studies have been performed on QCL devices. One recent study reported coherent oscillations attributed to resonant tunneling in a QC heterostructure for electron densities of up to several $10^{11} \mathrm{~cm}^{-2}$. The structure used in that experiment, however, was not an operating laser, and so does not directly apply to the dynamics under working conditions; to date, most information on the transport and relaxation in QCLs has been obtained indirectly through steady-state measurements.

Here, we report time-resolved mid-infrared (IR) degenerate pump-probe experiments on an operating QCL. The QCL was optimized for low threshold and high efficiency, and was not designed for the study of specific transport effects. The sample was based on one of the early four quantum-well active region designs. ${ }^{6}$ The device was processed into a ridge laser with a ridge width of $14 \mu \mathrm{m}$ and cavity length of $1.5 \mathrm{~mm}$ with facets left uncoated. As shown in Fig. 1, efficient tunneling injection from the injector ground state into the upper lasing state plays a key role in achieving population inversion and gain. As the electron leaves the active region, there is a transport delay through the superlattice injector before it is re-injected into the next period of active region.

For mid-IR pump-probe differential transmission (DT) measurements, tunable 200 fs mid-IR pulses were generated using an optical parametric amplifier and difference frequency generator (DFG), pumped by a $80 \mathrm{fs}, 250 \mathrm{kHz}$

\footnotetext{
${ }^{a)}$ Electronic mail: hyunyong@eecs.umich.edu.
}

Ti:sapphire regeneratively amplified laser. The DFG was tuned to be resonant with the QCL emission wavelength of $4.58 \mu \mathrm{m}$. The pump (800 pJ) and probe (40 pJ) beams were coupled into the QCL waveguide (with polarizations $\pm 45^{\circ}$ with respect to the lasing polarization, respectively), and the DT of the weak probe pulse was measured following the saturation of the gain by a perturbing pump pulse. A polarizer and a $10 \mu \mathrm{m}$ spatial filter were carefully positioned to isolate the signal coupled with the waveguide core. We estimate that approximately $1 \%$ of the pump and probe pulse energy was coupled into the waveguide core.

In Fig. 2(a), we show the DT signals for a series of bias at $78 \mathrm{~K}$. The DT signal is negative, corresponding to a pump-induced gain saturation and the decay of the DT corresponds to the gain recovery. The dynamics were fitted using the following rate-equation model; an example of the

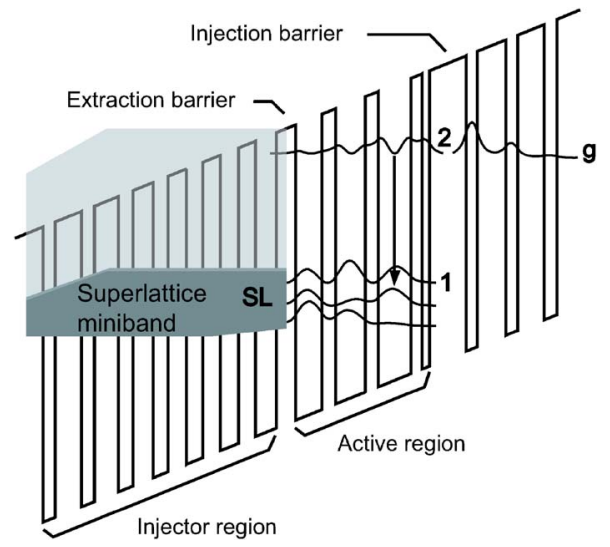

FIG. 1. (Color online) Calculated bandstructure of a portion of the active region and moduli squared of the relevant wavefunctions. An electric field of $68 \mathrm{kV} / \mathrm{cm}$ was applied to align the structure. The $\mathrm{In}_{0.61} \mathrm{Ga}_{0.39} \mathrm{As} / \mathrm{In}_{0.45} \mathrm{Al}_{0.55} \mathrm{As}$ layer sequence of one period of the active region, starting from the injection barrier is as follows: 46/9/14/ $41 / \mathbf{1 6} / 37 / \mathbf{1 7} / 33 / \mathbf{2 4} / 26 / \mathbf{2 1} / 23 / \mathbf{2 2} / 20 / \mathbf{2 3} / 19 / \mathbf{2 5} / 18 / \mathbf{3 0} / 16 / 33 / 15 / 35 / 14$. Thicknesses are in Angstrom, barrier layers are in bold, quantum-well layers are in roman, doped layers $\left(\mathrm{Si}, 2.65 \times 10^{11} \mathrm{~cm}^{-2}\right)$ are underlined. $g, 2,1$, and SL are the injector ground state, upper lasing state, lower lasing state, and superlattice state, respectively. The lasing wavelength was $4.58 \mu \mathrm{m}$ with the threshold current ranging from $171 \mathrm{~mA}$ at $10 \mathrm{~K}$ to $279 \mathrm{~mA}$ at $100 \mathrm{~K}$. 

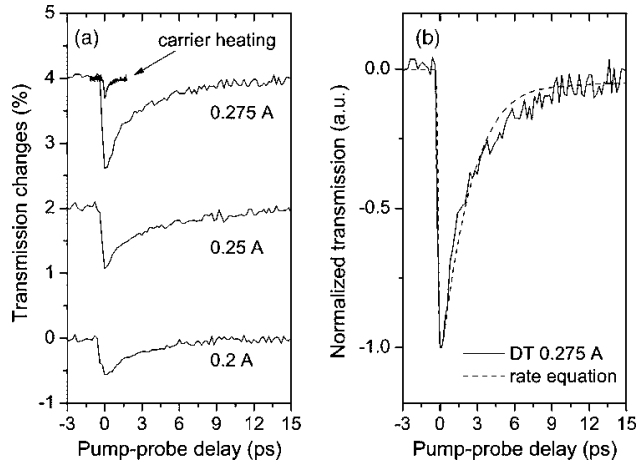

FIG. 2. (a) Bias dependent pump-probe DT signals measured at $78 \mathrm{~K}$. Contribution of carrier heating [transverse electric (TE) pump and transverse magnetic (TM) probe] is plotted on top of the DT at $275 \mathrm{~mA}$. The data are displaced for clarity. (b) Normalized DT signal at $275 \mathrm{~mA}$ bias current. The dashed line is the rate-equation fit.

rate-equation fit is shown in the Fig. 2(b) . The dynamics can also be equally fitted with two exponential decaying functions, as implied by this model. The rate equation reads

$$
\begin{aligned}
& \frac{d S}{d t}=\left[N_{p} \Gamma_{p} v_{g} g_{c}\left(n_{2}-n_{1}\right)-\frac{1}{\tau_{p}}\right] S+N_{p} \beta \frac{n_{2}}{\tau_{s p}}, \\
& \frac{d n_{g}}{d t}=\frac{n_{\mathrm{SL}}}{\tau_{\mathrm{SL}}}-\frac{n_{g}}{\tau_{\text {tunnel }}}, \\
& \frac{d n_{2}}{d t}=\frac{n_{g}}{\tau_{\text {tunnel }}}-\frac{n_{2}}{\tau_{2}}-\Gamma_{p} v_{g} g_{c}\left(n_{2}-n_{1}\right) S, \\
& \frac{d n_{1}}{d t}=\frac{n_{2}}{\tau_{2}}-\frac{n_{1}}{\tau_{1}}+\Gamma_{p} v_{g} g_{c}\left(n_{2}-n_{1}\right) S, \\
& \frac{d n_{\mathrm{SL}}}{d t}=\frac{n_{1}}{\tau_{1}}-\frac{n_{\mathrm{SL}}}{\tau_{\mathrm{SL}}},
\end{aligned}
$$

where $n_{g}, n_{2}, n_{1}, n_{\mathrm{SL}}$ are the injector ground state, upper lasing state, lower lasing state, superlattice electron density, and $S$ is the photon density. $N_{p}(=22)$ is the number of stages in the QCL, $\Gamma_{p}(1.6 \%$, calculated) is the confinement factor for one period, $v_{g}$ is the group velocity, $g_{c}$ [ $=4 \pi e^{2} z_{21}^{2} / \epsilon_{0} n \lambda(2 \gamma) L_{p}$ with $z_{21}=0.9 \mathrm{~nm}$ and $2 \gamma=20 \mathrm{meV}$ (Ref. 6)] is the gain cross section, $\tau_{p}$ is the photon lifetime, $\beta\left(=10^{-3}\right.$, assumed $)$ is the spontaneous emission factor, $\tau_{s p}$ is the spontaneous emission lifetime $(\simeq 1 \mu \mathrm{s}$, calculated $), \tau_{\mathrm{SL}}$ is the superlattice transport time, $\tau_{2}$ is the non-radiative lifetime of upper lasing state, $\tau_{1}$ is the non-radiative lifetime of lower lasing state, and $\tau_{\text {tunnel }}$ is the tunneling time from the injector to the upper lasing state.

Three notable features are observed in these measurements: (i) strong gain saturation near zero time delay, (ii) a "fast" initial partial gain recovery $(<1 \mathrm{ps})$, and (iii) a "slow" recovery $(2-3 \mathrm{ps})$. The fast gain recovery is attributed to the relaxation within the active region of a single stage of the $\mathrm{QCL}$, as the time scale is too short to arise from the transport between stages. The slow time constant corresponds to superlattice relaxation between adjacent stages, as shown below.

We now discuss these features in more detail. First, the partial depletion of the gain induced by the pump pulse near zero time delay gives rise to a negative probe DT signal Downloaded 28 Mar 2008 to 128.103 .60 .225 . Redistribution subje
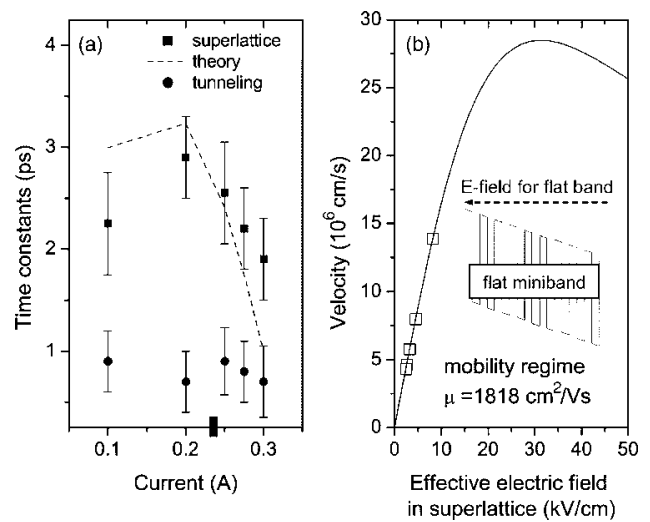

FIG. 3. (a) Gain recovery time constants at various bias currents. Threshold is indicated as a black tick mark. The filled squares correspond to the superlattice relaxation; the dashed line is the calculated dielectric relaxation in superlattice taking into account the inhomogeneous effective electric field at each bias current. The filled circles correspond to the resonant tunneling injection from the injector ground state and upper lasing state. The error bars are the chi squared determined from the rate-equation fits. (b) The empty squares are the superlattice velocities corresponding to the slow recovery components based on the serial circuit model and the solid line is the EsakiTsu velocity-field relation. The calculated electric field for a flat miniband condition was $\simeq 55 \mathrm{kV} / \mathrm{cm}$.

since a fraction of the electron population in the upper lasing state is transferred to the energetically lower lasing state by stimulated emission. Two photon or free carrier absorption may also contribute to this fast gain reduction through nonresonant carrier heating; to isolate these effects, we performed a DT measurement with TE-polarized pump and TMpolarized probe. The results, shown in Fig. 2(a), clearly demonstrate that the carrier heating effects play a minor role in the pump-induced gain compression.

Secondly, immediately following the gain saturation, a fast component $(<1 \mathrm{ps})$ for the gain recovery was observed at all bias conditions [Fig. 3(a)]. Two processes on the same time scale contribute to the fast recovery: carrier refilling from the injector ground state into the upper lasing state via tunneling and a double-phonon-like depopulation out of the lower lasing state. ${ }^{7}$

No gain recovery oscillations were observed under any bias conditions. Ideally, when there are no dephasing processes either due to homogeneous or inhomogeneous broadening (such as from interface roughness), the electronic wavepacket should oscillate coherently between the resonantly coupled injector ground state and upper lasing state with period of $\tau_{c o h}=2 \pi / \Omega_{R}$, where $\hbar \Omega_{R}$ is the energy splitting between the resonantly coupled states. ${ }^{5}$ When the dephasing rate is higher than the tunneling frequency, however, the coherent oscillations are suppressed and resonant tunneling proceeds as a rate process. For our laser, the calculated energy splitting at resonance $(8 \mathrm{meV})$ corresponds to an oscillation period of $517 \mathrm{fs}$. However, the time scale of electron-electron (e-e) scattering is calculated to be $<100 \mathrm{fs}$ (Refs. 8-11) for the electron densities present in the active region subband $\left(\sim 10^{10} \mathrm{~cm}^{-2}\right)$. Since this e-e scattering rate is larger than the energy splitting, it will contribute to a rapid decoherence in the resonant tunneling. Thus, the lack of coherent oscillation in this laser is to be expected.

Resonant tunneling in presence of dephasing has been investigated in detail previously. ${ }^{12}$ When the dephasing rate $1 / T_{2}$ due to scattering is short compared to the tunnel splitting $\hbar \Omega_{R}$, the tunneling rate $1 / \tau_{\text {tunneling }}$ can be written as to AlP license or copyright; see http://apl.alp.org/apl/copyright.jsp 


$$
\frac{1}{\tau_{\text {tunneling }}}=\frac{\Omega_{R}^{2} \frac{1}{2}\left(\frac{1}{T_{2}}\right)}{\left(\frac{1}{T_{2}}\right)^{2}+\left(\frac{\Delta E}{\hbar}\right)^{2}},
$$

where $1 / \tau_{\text {tunneling }}$ is the fast component observed in our experiments and $\Delta E$ is the energy detuning between the two coupled states. This model applies even when the upper subband decays due to stimulated emission. Resonant tunneling in presence of dephasing collisions results in a rate-equation limit if the dephasing rate is faster than the tunnel rate. Thus, for the optimal injection of electrons in an operating laser, it should be near the condition for "critical damping" so that no coherent oscillations will occur, as is the case in our QCL.

A similar model for the damping of the coherent tunneling has been developed by Williams ${ }^{13}$ The critical damping condition corresponds to a dephasing time of 41 fs. For dephasing faster than $41 \mathrm{fs}$, the coherent oscillation is overdamped as is the case in our QCL; this is a resonable condition for usual mid-IR QCL. ${ }^{10,14}$

Finally, the filled squares in Fig. 3(a) correspond to a slow gain recovery component with a time constant of 2-3 ps. We attribute this component to the superlattice transport. Following the pump-induced stimulated transition, electrons tunnel out of the lower lasing state into the superlattice, impulsively perturbing the equilibrium superlattice electron density. If the transport through the superlattice can be characterized by a perpendicular mobility within the miniband (low field regime), the equilibrium density profile across the superlattice will be restored in the dielectric relaxation time $t_{d}$, which may be written as $t_{d}=\epsilon / \sigma=\epsilon / q \mu N$, where $\epsilon$ is the background dielectric constant, $\sigma$ is the conductivity, $\mu$ is the mobility for perpendicular transport, and $N$ is the equilibrium density in the superlattice.

Because there is a significant electric field across the superlattice in an operating QCL, the density profile in the superlattice is nonuniform and the description of dielectric relaxation in the superlattice is a nontrivial problem. Nevertheless, if the field is low enough that the transport occurs in the mobility regime, we may apply an approximate model to understand the essential features. We first estimated the spatially varying density as follows. We modeled the QCL as a serial circuit comprised of two coupled resistors: the active region and the superlattice injector region. The resistance of each region is proportional to the lifetime of the carriers, which is determined from the DT dynamics. Thus the biasdependent voltage drops across the active and superlattice injector region, i.e., the effective electric field distributions can be obtained experimentally. Note that the effective electric field seen by electrons in the superlattice was obtained by subtracting the electric field for a flat miniband from the total field[see the inset of Fig. 3(b)]. An ensemble MonteCarlo simulation was then used to estimate the equilibrium electron densities in the superalattice subbands. The density in the upper part of the superlattice is the density relevant to the dielectric relaxation time; this is found to be only $1-5 \%$ of the doping density. Also from the bias-dependent effective electric field distributions, we used the Esaki-Tsu velocityfield relation ${ }^{15}$ to estimate the superlattice mobility and to verify that the transport is in the low-field regime [Fig. 3(b)].
The resulting bias-dependent dielectric relaxation time is shown as the dashed curve in Fig. 3(a). The electron velocity determined from this model is in the range of $10^{6} \mathrm{~cm} / \mathrm{s}$, which is consistent with previously reported Bloch transport in a superlattice miniband. ${ }^{4}$ Of course, a rigorous full quantum mechanical calculation would be required, where scattering through the superlattice subbands, hot carrier cooling, ${ }^{16}$ and dielectric relaxation are all considered on an equal footing.

For a self-consistency check, we calculated the superlattice mobility and effective mass based on the QCL design parameters. Given the miniband width $(\simeq 150 \mathrm{meV})$ and the estimated in-plane scattering time $(\simeq 42 \mathrm{fs})$ giving the best fit in Fig. 3, the calculated mobility corresponds to $\simeq 1800 \mathrm{~cm}^{2} / \mathrm{V} \mathrm{s}^{9}$. The corresponding band-edge effective mass for perpendicular transport in the superlattice was calculated to be $\simeq 0.04 m_{0}$, consistent with values found in other work [see Eq. (9) in Ref. 9].

In conclusion, we report time-resolved dynamics of resonant tunneling and superlattice transport in an operating QCL. Strong stimulated emission by the pump pulse produces a transient decrease of the gain in the active region. Gain compression due to carrier heating is shown to be comparatively small. Bias dependent recovery dynamics shows that the resonant tunneling is incoherent due to the strong dephasing in the active subband. On a longer time scale (few ps) the gain recovers to its steady-state value due to dielectric relaxation in superlattice.

${ }^{1}$ J. Faist, F. Capasso, D. L. Svico, C. Sirtori, A. L. Hutchinson, and A. Y. Cho, Science 264, 553 (1994).

${ }^{2}$ S. Lutgen, R. A. Kaindl, M. Woerner, T. Elsaesser, A. Hase, H. Künzel, M. Gulia, D. Meglio, and P. Lugli, Phys. Rev. Lett. 77, 3657 (1996); S. Lutgen, R. A. Kaindl, M. Woerner, T. Elsaesser, A. Hase, and H. Künzel, Phys. Rev. B 54, R17343 (1996).

${ }^{3}$ R. A. Kaindl, S. Lutgen, M. Woerner, T. Elsaesser, B. Nottelmann, V. M. Axt, T. Kuhn, A. Hase, and H. Künzel, Phys. Rev. Lett. 80, 3575 (1998); R. A. Kaindl, M. Wurm, K. Reimann, M. Woerner, T. Elsaesser, C. Miesner, K. Brunner, and G. Abstreiter, Phys. Rev. B 63, 161308 (2001).

${ }^{4}$ B. Deveaud, J. Shah, T. C. Damen, B. Lambert, and A. Regreny, Phys. Rev. Lett. 58, 2582 (1987).

${ }^{5}$ F. Eickemeyer, K. Reimann, M. Woerner, T. Elsaesser, S. Barbieri, C. Sirtori, G. Strasser, T. Müller, R. Bratschitsch, and K. Unterrainer, Phys. Rev. Lett. 89, 047402 (2002).

${ }^{6}$ J. Faist, M. Beck, T. Aellen, and E. Gini, Appl. Phys. Lett. 78, 147 (2001); J. Faist, D. Hofstetter, M. Beck, T. Aellen, M. Rochat, and S. Blaser, IEEE J. Quantum Electron. 38, 533 (2002).

${ }^{7}$ Details of the phonon-limited depopulation dynamics will be treated in a separate publication.

${ }^{8}$ W. H. Knox, D. S. Chemla, G. Livescu, J. E. Cunningham, and J. E. Henry, Phys. Rev. Lett. 61, 1290 (1988).

${ }^{9}$ F. Capasso, K. Mohammed, and A. Cho, IEEE J. Quantum Electron. QE-22, 1853 (1986)

${ }^{10}$ C. Sirtori, F. Capasso, J. Faist, A. Hutchinson, D. L. Sivco, and A. Y. Cho, IEEE J. Quantum Electron. 34, 1722 (1998).

${ }^{11}$ J. K. Jain and S. Das Sarma, Phys. Rev. B 36, 5949 (1987); S.-C. Lee and I. Galbraith, ibid. 59, 15796 (1999).

${ }^{12}$ K. Leo, J. Shah, J. P. Gordon, T. C. Damen, D. A. B. Miller, C. W. Tu, and J. E. Cunningham, Phys. Rev. B 42, 7065 (1990); S. A. Gurvitz, I. BarJoseph, and B. Deveaud, ibid. 43, 14703 (1991).

${ }^{13}$ B. Williams, Ph.D. thesis, Massachusetts Institute of Technology, 2003.

${ }^{14}$ T. Aellen, M. Beck, N. Hoyler, M. Giovannini, J. Faist, and E. Gini, J. Appl. Phys. 100, 043101 (2006).

${ }^{15}$ L. Esaki and R. Tsu, IBM J. Res. Dev. 89, 61 (1970).

${ }^{16}$ M. Woerner, K. Reimann, and T. Elsaesser, J. Phys.: Condens. Matter 14, R25 (2004). 